\title{
Secured Vehicle Life Cycle Tracking Using Blockchain and Smart Contract
}

\author{
Srinivasan Ananthanarayanan Bragadeesh and Arumugam Umamakeswari ${ }^{*}$
}

\author{
School of Computing, SASTRA Deemed University, Thanjavur, 613401, India \\ *Corresponding Author: Arumugam Umamakeswari. Email: aumamakeswari1967@gmail.com
}

Received: 27 April 2021; Accepted: 30 May 2021

\begin{abstract}
Life Cycle Tracking (LCT) involves continuous monitoring and analysis of various activities associated with a vehicle. The crucial factor in the LCT is to ensure the validity of gathered data as numerous supply chain phases are involved and the data is assessed by multiple stakeholders. Frauds and swindling activities can be prevented if the history of the vehicles is made available to the interested parties. Blockchain provides a way of enforcing trustworthiness to the supply chain participants and the data associated with the various actions performed. Machine learning techniques when combined decentralized nature of blockchains can be used to develop a robust Vehicle LCT model. In the proposed work, Harmonic Optimized Gradient Descent and Łukasiewicz Fuzzy (HOGDLF) Vehicle Life Cycle Tracking in Cloud Environment is proposed and it involves three stages. First, the Progressive Harmonic Optimized User Registration and Authentication model is designed for computationally efficient registration and authentication. Next, for the authentic user, the Gradient Descent Blockchain-based SVM Data Encryption model is designed with minimum CPU utilization. Finally, Łukasiewicz Fuzzy Smart Contract Verification is performed with encrypted data to ensure accurate and precise fraudulent activity deduction. The experimental analysis shows that the proposed method achieves significant performance in terms of life cycle's prediction time, overhead, and accuracy for a different number of users.
\end{abstract}

Keywords: Vehicle life cycle tracking; progressive harmonic optimization; blockchain; gradient descent; Łukasiewicz fuzzy; smart contract

\section{Introduction}

Blockchain can be described as a collection of ledgers organized into blocks that are linked with each other and are made strongly resistant to alteration by protecting it using cryptography. Each block contains details about the transaction that takes place between the users in a business network and are linked together to form a chain of records, which is stored by every user. All transactions are cryptographically signed by the participants and verified. This public distributed ledger prevents any fraudulent action as each user in the network has a copy of the ledger and can detect any discrepancy easily. The blocks are validated and added into the blockchain by the miners by solving a complex mathematical problem also referred to as consensus algorithms. The whole structure is made self-governing by the use of smart contracts and

This work is licensed under a Creative Commons Attribution 4.0 International License, which permits unrestricted use, distribution, and reproduction in any medium, provided the original work is properly cited. 
doesn't involve any intermediate third party. Smart contracts are self-executable code constructs that are triggered based on conditions defined during the creation of the blockchain.

Blockchain has been increasingly adopted in supply chains [1]. They create a secure medium for the exchange of records and provide transparency throughout the lifecycle. This decentralized system makes the process much easier as it is not controlled by a single entity. The peer-to-peer system is also fast and has no single point of failure. The intrinsic characteristics of blockchain have fascinated several real-time applications and specific applications in vehicle life cycle tracking. Recently, many organizations have taken a keen interest in engaging blockchain for developing models in vehicle LCT.

In [2], a blockchain-based framework was designed for tracking vehicles in a significant manner that embodies the managing of trust between parties, designing a transparent system, and providing a significant access control mechanism. Also, blockchain was tailor-made to allow user control for auxiliary transactions, involving inspection, renting, and ensuring insurance. In the blockchain-based framework, the usage control model was merged with the respective IoT devices to monitor the vehicle continuously depending on certain conditions, and if required remote access of revoking was also ensured. Finally, a comprehensive transaction set was also documented over an immutable ledger that bestowed trust, transparency, and an absolute record archive, therefore ensuring throughput and latency. Despite improvement observed in throughput and latency, the vehicle tracking time was not focused. To address this issue, a Progressive Harmonic Search Optimization (PHSO) algorithm is proposed that with the aid of an optimization model generates a computationally efficient key for each registered user.

A deep feature-based objective tracking method was proposed in [3] for efficient tracking of vehicles. A vehicle tracking method based on a Detection-Based Tracking (DBT) framework for traffic scenes is presented. The You Only Look Once (YOLO) model is used to design the model of vehicle detection, and two constraints including object attribute information and intersection over union (IOU), are combined to modify the vehicle detection box. Vehicle detection precision is improved by this approach. The work involved two processes, object detection, and object tracking. First, a detection box merge strategy was utilized to block the device from detecting an object more than once or partially detecting an object. Next, object tracking was performed by applying a deep feature-based Correlation Filter tracker. With this, the object location was tracked efficiently for the successive frame. Moreover, the quality of the object being tracked was measured based on the peak to sidelobe ratio (PSR). The proposed technique achieves improvement in throughput and latency, but the vehicle tracking time was not focused. The improvement in success rate is obtained, whereas the overhead and accuracy with which the vehicle LCT was said to be acquired remained unaddressed. This method did not use any Cryptographic algorithms for ensuring the security of the data. The proposed technique focuses on reducing the tracking time and memory overhead improves the detection accuracy and employs machine learning-based technique for securing the data. This provides better performance than the techniques proposed by the above two works.

Besides, with the trackers possessing a poor quality of object being tracked in numerous successive frames, a spatial location threshold was employed to rectify the forecasted locations. With these two-stage processes, the proposed multi-object tracking algorithm was identified to be competent in regularly and endlessly tracking objects with a higher success rate. Despite improvement obtained in success rate, the overhead and accuracy with which the vehicle LCT was said to be acquired remained unaddressed. To concentrate on this issue, Gradient Descent Blockchain-based Data Encryption (GDB-DE) with the aid of Lukasiewicz Fuzzy ensures minimum overhead with accurate tracking.

The major contributions of this research work can be summarized below.

- A Progressive Harmonic Search Optimization (PHSO) algorithm is proposed for efficient user registration and authentication 
- A Gradient Descent Blockchain-based Data Encryption (GDB-DE) algorithm is proposed for performing encryption and decryption of data with minimum memory overhead and improved CPU utilization

- Lukasiewicz Fuzzy Smart Contract (LFSC) algorithm is proposed for verification to ensure security and provide a mechanism to detect any malicious activity.

The rest of the paper is structured as follows. Section 2 briefs the background technologies on the blockchain and vehicle life cycle tracking systems. Section 3 introduces the proposed machine learning technique for implementing a vehicle life cycle tracking system. Section 4 provides the experimental settings followed by an in-depth discussion of the various results obtained. Section 5 concludes the paper.

\section{Related Works}

LCT is extensively utilized for estimating the environmental influences of a product or service. Gathering genuine data is a vital ultimatum in LCT due to the intricacies entangled in tracking and assessing inputs and outputs at numerous supply chain phases. Blockchain technology proves to be a viable solution to control the issues involved in supply chain management. A comprehensive ecosystem of blockchain reviewing its applications and problems faced was discussed in detail in [4]. Yet another system architecture combining blockchain, IoT, and big data was implemented in [5].

Security and privacy preservation are said to be the key to an electronic government system. At the same time, information security and privacy are improved by blockchain technology where the data is stored in an encrypted manner and can also be distributed globally over the entire network. A framework for decentralized e-government Peer-to-Peer (P2P) system utilizing blockchain technology was proposed in [6] for ensuring the security and privacy of data while improving trust. Supply Chain Management (SCM) involves financial, material, and information flow management between Supply Chain (SC) members. Several research works have been conducted with this regard in designing supply chain cooperation, combination, and associated subject modes with streaming techniques between several economic institutions to enhance the comprehensive execution of SC.

With the increase in the number of vehicles, there is a corresponding increase in the issues associated with the vehicle such as its authenticity, history, etc. Vehicle-related fraud activities in Europe [7], like the mileage fraud, were found to be prevalent in $30 \%$ of the car sales history. This leads to several doubts among the consumers decreasing trust in sales. Another major concern is the putting of wrecked cars into use which becomes a major threat to the safety of the vehicle users. Counterfeit spare parts [8], create distrust among all brands even which work genuinely. With the blooming interest towards the autonomous vehicle, there also exist privacy concerns associated with the data generation and usage from the IoT sensors $[9,10]$ fixed in the vehicles. Blockchain can benefit in various areas associated with the vehicle such as vehicle communication, vehicle forensics, and insurance [11]. Blockchain incorporated in ad hoc networks [12] can form an evident part of the smart cities by improving the large-scale vehicles' network. Even for several maintenance processes in the industries [13], smart contracts can be used to automate a process when an event occurs, making jobs easier for the stakeholders involved. The authors in [14] provide an overview of all activities happening in a production unit of the industry by incorporating blockchain technology in the car supply chain along with IoT technology. This can help to simplify the process of maintenance and improves collaboration. Distributed ledgers are used to store data so that the history of the product is available and hence increase traceability.

In [15], a scalable life cycle inventory model was designed to maintain data usability over time. A twoway authentication and key agreement algorithm using encryption and signature model were proposed in [16]. Yet another strong modular approach with common transport and vehicle characteristics as building 
blocks for virtual vehicles was designed in [17]. A product life cycle supply chain model based on blockchain system cost chain scenario was presented in [18] via revenue-sharing contract. Though life cycle time was reduced, however, tracking success rate was not analyzed. Accurate multiple vehicle detection in a complex traffic domain is too cumbersome. Moreover, this procedure is made more tedious when there is overlapping of vehicles. In [19], Enhanced Convolution neural network with Support Vector Machine (ECNN-SVM) based vehicle detection was proposed for efficient computation. In [20], a life cycle sustainability assessment was proposed for ensuring smooth tracking in self-driven heavy-duty trucks.

In [21] a technique was proposed to avoid the insurance frauds made by the vehicle owners by assisting the insurance companies to manage their policies. The double-dipping problem is addressed by claim validation made using smart contracts and recording the lifecycle of a vehicle in the blockchain. To avoid odometer fraud in vehicles, a blockchain architecture is proposed in [22]. PBFT (Probabilistic Byzantine Fault Tolerance) algorithm is incorporated which helps in faster block generation and as master nodes are available, it ensures there are no invalid blocks. The authors in [23] propose an anonymous authentication with selective revocation where the privacy of the user details is preserved. A blockchain-based anonymous authentication protocol is used where multiple attributes of the user can be preserved and it can also allow selective revocation of the details. Verifiers verify the details of the user and it is then put into the blockchain.

Efficient management of traffic with the aid of cameras that have been mounted on drones or airplanes in recent years has started becoming a major topic of discussion. Airborne surveillance provides numerous advantages in comparison with conventional monitoring techniques. Such methods are found to be bestowing a large amount of coverage with minimum expenses and are also found to be comparatively better in case of emergencies. In [24], multi-criterion factors were analyzed to minimize life cycle cost. Yet another robust real-time approach utilizing top-hat and bottom-hat transformation using morphological operation was proposed in [25]. Structural Kalman filter was applied in [26] for enhancing detection and vehicle tracking to a greater extent.

In general, most of the relevant studies have certain drawbacks in time, accuracy, error rate, or privacy. Combining the advantages and drawbacks of the abovementioned existing methods, this work proposes a secured and optimized system based on blockchain. By incorporating blockchain, the security of data can be increased. With multiple parties involved in various stages of a vehicle lifecycle, there are high chances for data manipulation. The use of blockchain can make the process of vehicle lifecycle management better by providing reliability and accountability. It provides a source of immutable data with any data updates only being performed by authorized personnel. The proposed method uses the open, self-organized, tamper-proof, and distributed characteristics of blockchain technology to design Harmonic Optimized Gradient Descent and Łukasiewicz Fuzzy (HOGD-LF) Vehicle Life Cycle Tracking in Cloud Environment which improves the life cycle prediction time, accuracy, and the overall overhead.

\section{Methodology}

There are a lot of opportunities to incorporate blockchain in the vehicle lifecycle to elevate the automotive industry. In the manufacturing process, the status of the vehicle assembly can be correctly reported. Then comes the manual data entry part where the vehicle parameters are entered which is validated automatically in the blockchain. Even for vehicle transfer, history could be verified in blockchain ensuring transparency. If the vehicle is under insurance, the vehicle profile can be verified, in case of accidents before granting claims. Blockchain has the potential to enforce trust and also provide opportunities for collaboration among the stakeholder. It also provides transparency among the users. Decentralized systems make it easier to enforce security by eliminating single authority ownership and single storage. It also simplifies the maintenance process. 
Vehicle life cycle tracking in the automotive industry has encountered many years of evolution, which has brought out considerable changes for improved tracking. Despite its evolution, due to the extreme competition, fraudulent activities are said to take place either during the purchase or in the regional transport office, or at the insurance claim stage. Several research works have suggested that blockchain technology can be used to secure data between stakeholders. Blockchain enables a decentralized platform for managing data that in turn permits multiple stakeholders to update, manage and perform data verification in a concurrent manner. In this work, a Harmonic Optimized Gradient Descent and Łukasiewicz Fuzzy (HOGD-LF) Vehicle Life Cycle Tracking in Cloud Environment is proposed. Initially, a system model for the proposed framework in the cloud environment is provided, which is followed by a description of various techniques used in the proposed HOGD-LF.

\subsection{System Model}

Manufacturers (M) produce and develop vehicles. Once the product is manufactured, it is sold to the buyer (B) who uses it and maintains it by taking it to service whenever needed. Buyers are the people who purchase the product from the manufacturers. They are the customers for whom the product is mainly made. Good interaction between the producer and customer can often lead to well-established relationships which prove to be useful in further sales. Transport Authority (TA) checks the standards of the vehicle. They govern the processes such as registration, maintenance, emission check, vehicle tax, etc. TAs have an overview of the all processes associated with the vehicle. They test and verify the vehicles based on safety standards. Before releasing a model, the manufacturer has to get a nod from the TA stating that it has been made in compliance with the standards and norms.

Whenever there is a sale of a used vehicle, the history of the vehicle is often not fully available to the buyer. Several aspects such as repairs, accidents, emissions, and safety level are often not fully known to the customers. Reports made by the seller may even not be fully genuine. It can also be the case for Insurance claims. In such cases, the history of transactions can be easily accessed through the blockchain by the buyer to verify details.

The quality check report provided by the regulatory boards after checking a vehicle may also be updated into the network and help the buyer maintain the vehicle standards throughout its usage. It also makes it easier for the investigation when there are any problems associated with the vehicles. There are many parties involved throughout the lifecycle of a vehicle and collaboration would prove to be an extremely useful factor for enhancing the vehicle-related services. The key to successful management lies in the interactions among them and low operating costs.

The vehicle lifecycle network provides provisions for different transactions. These include buying, registration, getting insurance, and requesting claims during accidents, reselling of used vehicles, and disposal. All these transactions have to be recorded into the blockchain network to enable traceability and consistency. The blockchain network is formed by the nodes or users (Buyers, Manufacturers, Transport Authority, and insurance agent), a network protocol, and data structure. Inside the network, nodes of the blockchain perform various transactions in a verified manner (i.e., registration/user authentication, data encryption, and verification). The network protocol is responsible for carrying out the verification process and consensus across nodes in the blockchain network. Finally, the data structure of blockchain refers to an index of blocks that are linked together, where each block records the transactions in the network.

\subsection{Harmonic Optimized Gradient Descent and Lukasiewicz Fuzzy (HOGD-LF) Vehicle Life Cycle} Tracking

In this section, an overview of the proposed Harmonic Optimized Gradient Descent and Lukasiewicz Fuzzy (HOGD-LF) method for Vehicle Life Cycle Tracking is presented. Fig. 1 illustrates the developed 
Blockchain architecture for vehicle life cycle tracking in the cloud environment. Initially, the user will register to the cloud service provider and receive the key for data encryption using Progressive Harmonic Search Optimization (PHSO) algorithm. The user will then authenticate with the aid of a user id, password, and a random number. Next, upon successful authentication, the authenticated user can upload their encrypted data to the cloud. For encryption, we implement Gradient Descent Blockchain-based Data Encryption (GDB-DE) algorithm. The encrypted data is stored in the cloud via a cloud service provider (CSP). The CSP at this juncture creates a block for each data that is uploaded by the user. In the end, the Insurance Authority (IA) can collect the claim details and the different updates performed on those claims from the blockchain and performs verification. The experimental evaluation discloses that the proposed method has acquired better performance by reducing time and increasing the accuracy. A detailed description of the proposed method is given in the forthcoming sections.

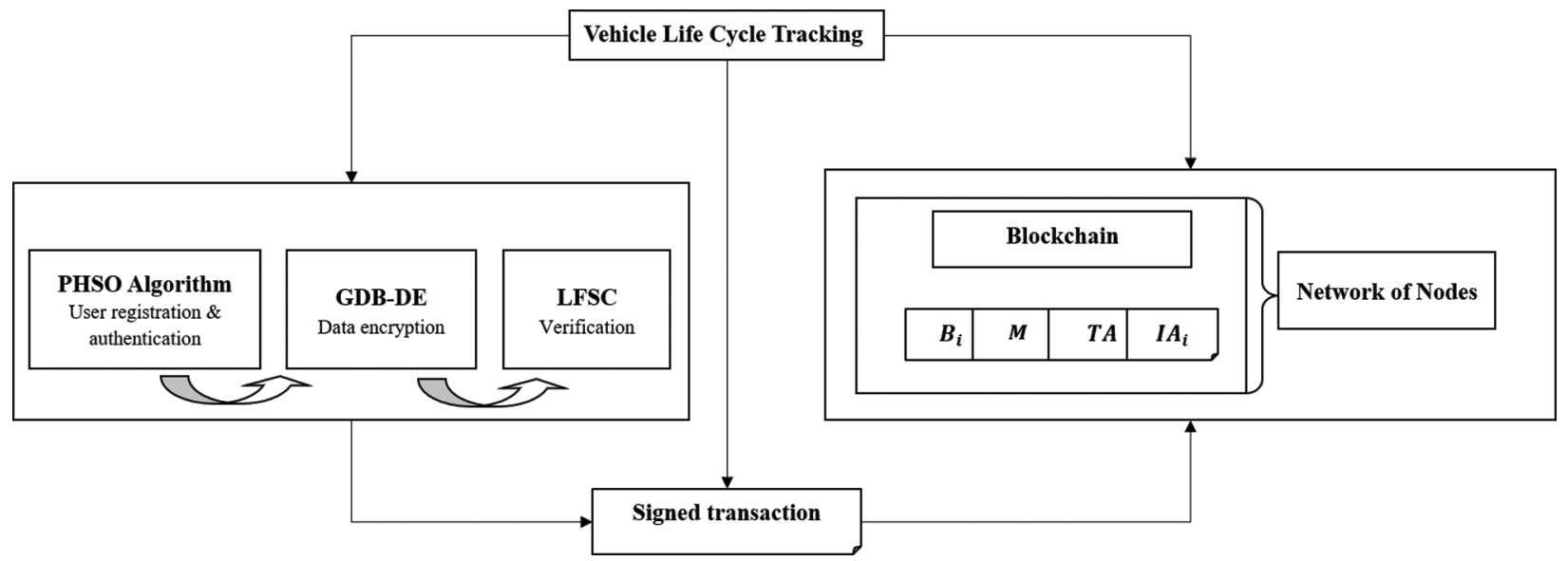

Figure 1: Block diagram of harmonic optimized gradient descent and Łukasiewicz fuzzy (HOGD-LF) method

\subsection{Progressive Harmonic Optimized User Registration and Authentication Model}

In our proposed method, the user (i.e., buyer ' $\mathrm{B}_{\mathrm{i}}$ ', manufacturer ' $\mathrm{M}$ ', insurance agent ' $\mathrm{IA}_{\mathrm{i}}$ ', transport authority 'TA') will in the first instance carry out the registration procedures with the legal authority ('TA'). The Progressive Harmonic Search Optimization (PHSO) algorithm is used for authorization purposes. Once the user has successfully registered, a key ' $\mathrm{K}=\mathrm{U}_{\mathrm{i}}\left[\mathrm{k}_{\mathrm{i}}\right]$ ' is provided. These credentials are necessary for the user to participate in the vehicle life cycle tracking process. Fig. 2 shows the block diagram of the Progressive Harmonic Optimized User Registration and Authentication model.

As illustrated in Fig. 2, the process starts by defining an initial Progressive Harmonic Matrix (PHM) that comprises a definite number of progressively initiated solutions to the optimization issues under consideration. For ' $m$ ' issues, a PHM with the magnitude of ' $n$ ' is denoted as follows.

$\mathrm{PHM}=\left[\begin{array}{cccc}\mathrm{C}_{1}^{1} & \mathrm{C}_{2}^{1} & \ldots & \mathrm{C}_{\mathrm{n}}^{1} \\ \mathrm{C}_{1}^{2} & \mathrm{C}_{2}^{2} & \ldots & \mathrm{C}_{\mathrm{n}}^{2} \\ \ldots & \ldots & \ldots & \ldots \\ \mathrm{C}_{1}^{\mathrm{m}} & \mathrm{C}_{2}^{\mathrm{m}} & \ldots & \mathrm{C}_{\mathrm{n}}^{\mathrm{m}}\end{array}\right]$

From the above matrix (1), ' $C_{1}^{\mathrm{i}}, C_{2}^{\mathrm{i}} \ldots \mathrm{C}_{\mathrm{n}}^{\mathrm{i}}$ ' corresponds to the solution candidate for vehicle life cycle tracking registration. The next step is to obtain a new solution ' $\mathrm{C}_{1}^{\prime}, C_{2}^{\prime} \ldots \mathrm{C}_{\mathrm{n}}^{\prime}$ '. Each component (i.e., dealer ' $\mathrm{D}$ ') of this solution is assigned with the respective buyer ' $\mathrm{B}$ ' based on Harmony Memory Considering Rate ('HMCR'), where 'HMCR' refers to the probability of assigning a dealer ' $\mathrm{D}$ ' with the 
respective buyer 'B'. The progressive 'HMCR' (i.e., enhanced candidate solution) employed in our work is mathematically expressed as given below.

PHMCR $=\mathrm{HMCR}_{\text {crest }}\left[\frac{\ln \left(\frac{\mathrm{HMCR}_{\text {base }}}{\mathrm{HMCR}_{\text {crest }}}\right)}{\operatorname{Max}_{\mathrm{NI}}}\right] * \mathrm{NI}$

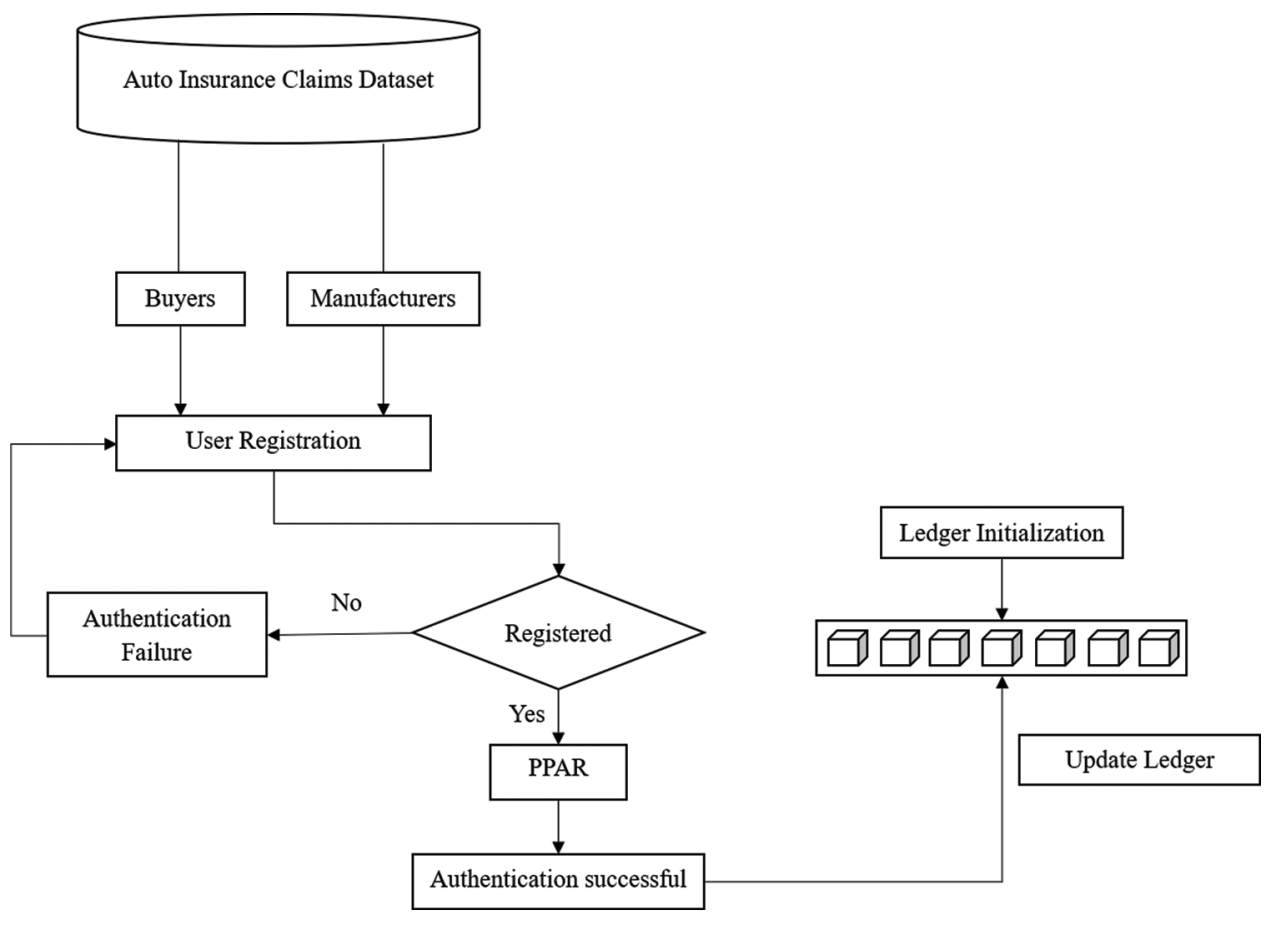

Figure 2: Block diagram of progressive harmonic optimized user registration and authentication model

The Progressive HMCR (PHMCR) is obtained based on the logarithmic value of the ratio of base HMCR value, crest HMCR value, and the maximum number of allowable iterations respectively as shown in Eq. (2). Another important factor of consideration during the registration process is the Pitching Adjust Rate (PAR) that also refers to the probability of a candidate (i.e., registered buyer assigned to a corresponding registered dealer). In our work, the Progressive PAR (PPAR) is utilized for progressive assignment and is mathematically evaluated as given below.

$\operatorname{PPAR}(\mathrm{NI})=\mathrm{PAR}_{\text {base }}+\frac{\mathrm{PAR}_{\text {crest }}-\mathrm{PAR}_{\text {base }}}{\mathrm{MAX}_{\mathrm{NI}}} * \mathrm{NI}$

From Eq. (3) the progressive PAR for different numbers of iterations 'PPAR(NI)' is evaluated based on the crest of PAR, the base of PAR and the maximum number of iterations ' $M A X_{\mathrm{NI}}$ ' respectively. The third step is updating the PHM, wherein the new solution obtained from the above step is used. In our work, the user provides a unique id 'UID', password 'PWD', and a random number ' $\mathrm{U}_{\mathrm{i}}(\mathrm{RNo})$ ' for authentication. When a better fitness than that of the worst member in the PHM is obtained, PHM is updated accordingly and the authentication of the user is acknowledged. If the fitness value obtained is not suitable, the corresponding request for user authentication is rejected. The pseudo-code representation of the 
Progressive Harmonic Search Optimization (PHSO) algorithm is given below.

Algorithm 1: Progressive Harmonic Search Optimization (PHSO) algorithm

Input: User ' $\mathrm{U}=\mathrm{B}, \mathrm{M}$, TA, IA, D', Buyer ' $\mathrm{B}=\mathrm{B}_{1}, \mathrm{~B}_{2}, \ldots, \mathrm{B}_{\mathrm{n}}$ ' Manufacturers ' $\mathrm{M}=\mathrm{M}_{1}, \mathrm{M}_{2}, \ldots, \mathrm{M}_{\mathrm{n}}$ ', Transport Authority 'TA', Insurance Authority 'IA $=\mathrm{IA}_{1}, \mathrm{IA}_{2}, \ldots, \mathrm{IA}_{\mathrm{n}}$ ', Dealers ' $D=d_{1}, d_{2}, \ldots, d_{n}$ '

Output: Computationally efficient key ' $\mathrm{key}$ ' $\mathrm{K}=\mathrm{U}_{\mathrm{i}}\left[\mathrm{k}_{\mathrm{i}}\right]$ ' Generation time

1: Initialize PHM (as in Eq. (1)), unique id 'UID', password 'PWD', and a random number '(RNo)'

2: Begin

3: $\quad$ For each User ' $U$ '

4: $\quad$ Obtain enhanced candidate solution using Eq. (2)

5: $\quad$ Obtain progressive PAR using Eq. (3)

6: $\quad$ If ' $(\operatorname{rand}(0,1)<$ PPAR $)$ '

7: $\quad$ Let $\mathrm{C}_{\mathrm{i}}^{\text {new }}=\mathrm{C}_{\mathrm{i}}^{\text {new }}+\operatorname{rand}(-1,1) * \operatorname{PPAR}(\mathrm{NI})$

8: $\quad$ Else

9: $\quad$ Let ' $C_{i}^{\text {new }}=\mathrm{L}_{\mathrm{i}}+\operatorname{rand}(-1,1)$ '

10: $\quad$ End if

11: $\quad$ If ' $\mathrm{f}\left(\mathrm{C}_{\mathrm{i}}^{\text {new }}\right)<\mathrm{f}\left(\mathrm{C}_{\mathrm{i}}^{\text {worst }}\right)$ ' and 'VUID $=\mathrm{UID}$ ', 'VPWD $=\mathrm{PWD}$ ', 'VRNo $=\mathrm{RNo}$ '

12: $\quad$ User acknowledged with authentication

13: $\quad$ Else If ' $\mathrm{f}\left(\mathrm{C}_{\mathrm{i}}^{\text {new }}\right)>\mathrm{f}\left(\mathrm{C}_{\mathrm{i}}^{\text {worst }}\right)$ ' and 'VUID $\neq \mathrm{UID}$ ', 'VPWD $\neq \mathrm{PWD}$ ', 'VRNo $\neq \mathrm{RNo}$ '

14: $\quad$ User access rejected

15: $\quad$ End if

16: End for

17: End

As given in the above algorithm, the objective here remains in performing the registration and authentication process with a computationally efficient key generation time. This is achieved by applying a Progressive Harmonic Search Optimization (PHSO) algorithm. The use of progressive functions for both HMCR and PAR ensures computationally efficient key generation with a high degree of authentication.

\subsection{Gradient Descent Blockchain-Based SVM Data Encryption Model}

The authenticated user can upload or securely download the data. For encryption, we have assumed that the cloud service provider aims at training SVM models using the data collected from authentication users. SVM is a supervised learning model that gives the maximum-margin hyperplane to classify the test data. The hyperplane is expressed as given below.

$\mathrm{q}=\mathrm{w}^{\mathrm{T}} \mathrm{p}+\mathrm{b}, \quad\left(\mathrm{p}_{\mathrm{i}}, \mathrm{q}_{\mathrm{i}}\right) \in \mathrm{D}$

such that

$\left[\mathrm{w}^{\mathrm{T}} \mathrm{p}_{\mathrm{i}}+\mathrm{b} \geq 1\right]$, if $\mathrm{q}_{\mathrm{i}}=+1$;

$\left[\mathrm{w}^{\mathrm{T}} \mathrm{p}_{\mathrm{i}}+\mathrm{b} \leq 1\right]$, if $\mathrm{q}_{\mathrm{i}}=-1$ 
From Eq. (4), 'w' refers to normal vector (i.e., the users, buyers, manufacturer, insurance agent, and transport authority registered with the blockchain) to the plane, with ' $q$ ' representing either ' +1 ' or ' -1 ' each denoting the class to which ' $p_{i}$ ' (i.e., refers to the vehicle purchase class, registration with the transport authority class, insurance class) belongs to. The cloud service provider encrypts the data using their private keys and records them in a blockchain-based ledger. This encrypted data acquired from the Auto Insurance Claims dataset is uploaded into the cloud. The encrypted data from the user is stored in the cloud. Fig. 3 shows the block diagram of the Gradient Descent Blockchain-based SVM Data Encryption model.

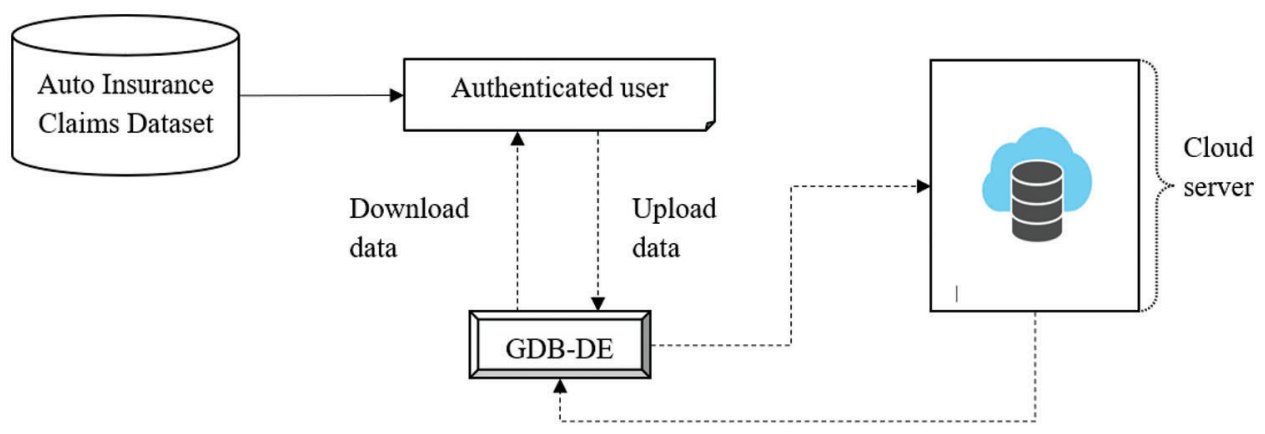

Figure 3: Block diagram of gradient descent blockchain-based SVM data encryption model

The user's data is encrypted by utilizing the Gradient Descent Blockchain-based Data Encryption (GDB-DE) algorithm and transfer to the cloud server. The GDB-DE refers to a Gradient Descent model that provides unsupervised learning encryption. It integrates a Gradient Descent function based on unsupervised learning using public key ' $\mathrm{PK}$ ' for encryption and private key ' $\mathrm{K}$ ' for decryption respectively.

The Gradient Descent model converts the conventional SVM technique into a loss minimization problem mathematically expressed as given below.

$\min \frac{1}{2}(\mathrm{w})^{2}+\mathrm{C} \sum_{\mathrm{i}=1}^{\mathrm{n}} \operatorname{Sum}\left\{0,1-\mathrm{q}_{\mathrm{i}}\left(\mathrm{wp}_{\mathrm{i}}-\mathrm{b}\right)\right\}$

In Eq. (5), ' $C \sum_{i=1}^{n} \operatorname{Sum}\left\{0,1-\mathrm{q}_{\mathrm{i}}\left(\mathrm{wp}_{\mathrm{i}}-\mathrm{b}\right)\right\}$ ' refers to the Sum-of-Hinge-Loss function with the misclassification value represented as ' $\mathrm{C}$ ' for respective classes ' $\mathrm{p}_{\mathrm{i}}$ '. Then the gradient measurement of SVM for corresponding encryption is mathematically expressed as given below.

$\nabla_{\mathrm{t}}=\lambda \mathrm{w}_{\mathrm{t}}-\sum\left[\mathrm{E}\left(\mathrm{wp}_{\mathrm{i}}+\mathrm{b}\right)\right] * \mathrm{p}_{\mathrm{i}} \mathrm{q}_{\mathrm{i}}$

From Eq. (6), gradient measurement of SVM ' $\nabla_{t}$ ' for respective classes ' $p_{i}$ ' is evolved encryption is performed based on the evidence function ' $E$ '. Correspondingly, the encrypted data for three different classes using quadratic function are given below as Eq. numbers (7)-(9).

$\left(\mathrm{f}_{\mathrm{i}}+\mathrm{f}_{\mathrm{j}}\right)=\left(\mathrm{f}_{\mathrm{i}}\right) *\left(\mathrm{f}_{\mathrm{j}}\right)\left(\bmod \mathrm{N}^{2}\right)$

$\left(\mathrm{f}_{\mathrm{i}}-\mathrm{f}_{\mathrm{j}}\right)=\left(\mathrm{f}_{\mathrm{i}}\right) *\left(\mathrm{f}_{\mathrm{j}}\right)^{-1}\left(\bmod \mathrm{N}^{2}\right)$

$\left(\mathrm{lf}_{\mathrm{i}}+\mathrm{mf}_{\mathrm{j}}\right)=\left(\mathrm{f}_{\mathrm{i}}\right)^{1} *\left(\mathrm{f}_{\mathrm{i}}\right)^{\mathrm{m}}\left(\operatorname{modN}{ }^{2}\right)$

In Eq. (9), '( $\left(\mathrm{f}_{\mathrm{i}}+\mathrm{mf}_{\mathrm{j}}\right)$ ' corresponds to quadratic function for features ' $\mathrm{f}_{\mathrm{i}}$ ', ' $\mathrm{f}_{\mathrm{j}}$ ', using ' $\operatorname{modN} \mathrm{N}^{2}$, modulus function. The pseudo-code representation of Gradient Descent Blockchain-based Data Encryption (GDB$\mathrm{DE}$ ) is given in algorithm 3. The objective here remains in performing the encryption and decryption. Here, the encryption data (i.e., features for example ' $f_{i}$ ', ' $f_{j}$ ') utilizing quadratic function is uploaded into the cloud using the public key whereas the decryption is performed using the private key respectively. 
The proposed GDB-DE algorithm enables the encryption of data by different users based on the features available in the data. The conversion of SVM into a lossless minimization function using the gradient descent model helps in reducing the overall memory overhead for tracking. The use of separate keys generated for encryption and decryption improves the security of the system and CPU utilization.

Algorithm 2: Gradient Descent Blockchain-based Data Encryption (GDB-DE) algorithm

Input: User ' $\mathrm{U}=\mathrm{B}, \mathrm{M}$, TA, IA, $\mathrm{D}$ ', Buyer ' $\mathrm{B}=\mathrm{B}_{1}, \mathrm{~B}_{2}, \ldots, \mathrm{B}_{\mathrm{n}}$ ' Manufacturer (different make) ' $\mathrm{M}=\mathrm{M}_{1}, \mathrm{M}_{2}, \ldots, \mathrm{M}_{\mathrm{n}}$ ', Transport Authority 'TA', Insurance Authority ' $\mathrm{IA}=\mathrm{IA}_{1}, \mathrm{IA}_{2}, \ldots, \mathrm{IA}_{\mathrm{n}}$ ', Dealer ' $D=d_{1}, d_{2}, \ldots, d_{n}$ ', feature ' $f$ ', Cloud Service Provider 'CSP'

Output: Encryption and decryption with minimum CPU utilization

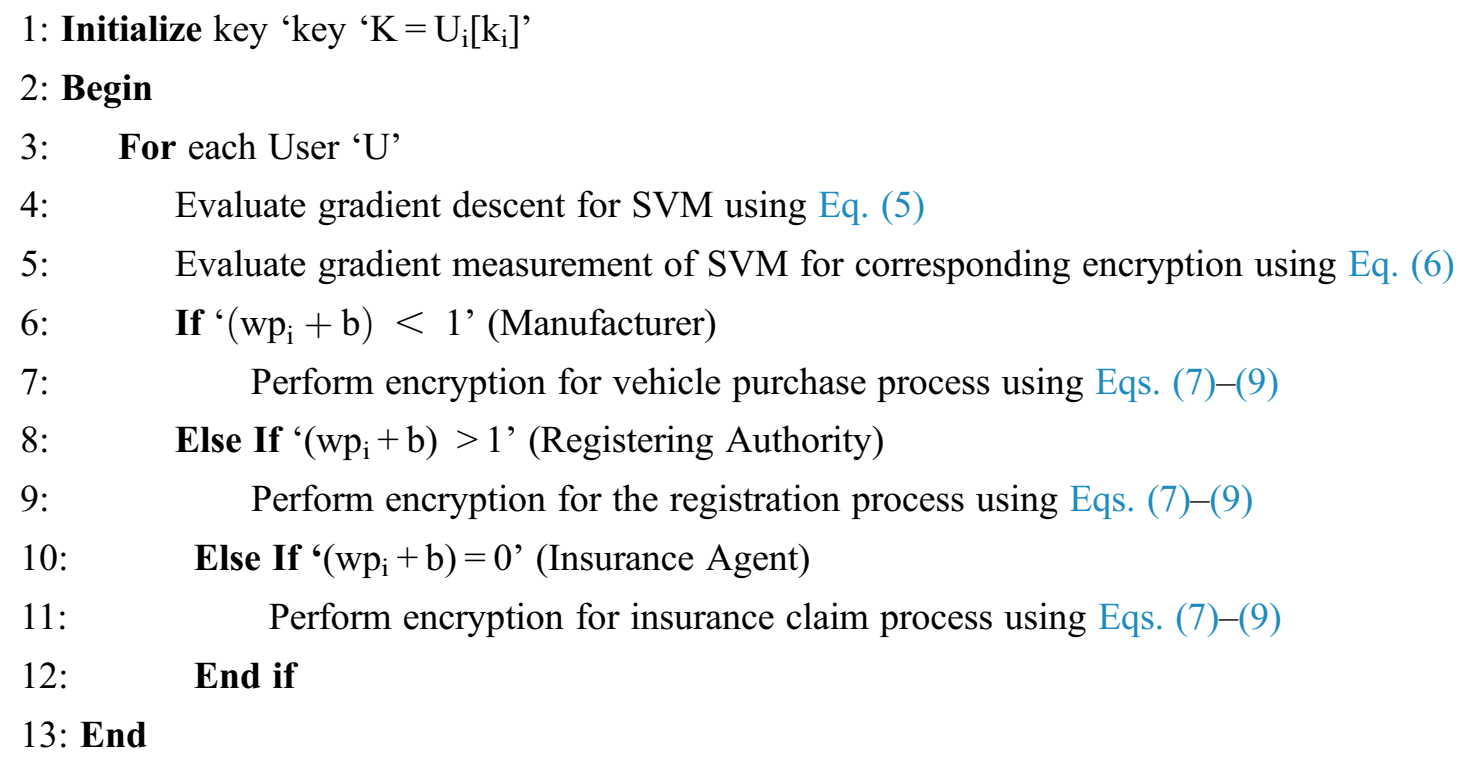

\subsection{Lukasiewicz Fuzzy Smart Contract Verification}

The encrypted data of all the users for vehicle life cycle tracking is stored in the cloud in the form of blocks in the blockchain network. The block here includes the transaction list along with the data, hash value of the preceding block, and the timestamp respectively. Łukasiewicz Fuzzy Smart Contract Verification model is used for vehicle life cycle tracking. Fig. 4 shows the block diagram of the Lukasiewicz Fuzzy Smart Contract Verification model. The objective behind the design of the Lukasiewicz Fuzzy Smart Contract algorithm is the conjunction (i.e., whereupon successful conditional checking made with the transport authority being true, buyers insurance claim with the respective insurance agent is predicted) in an accurate and precise manner. The pseudo-code representation of the Lukasiewicz Fuzzy Smart Contract is given below.

As given in the Łukasiewicz Fuzzy Smart Contract algorithm (Algorithm 3), the objective remains in performing data accesses, data modification in an accurate and precise manner. Moreover, information of user accessing the information, the action being performed, place of access is also stored in the respective block. Thus, our proposed method is said to ensure security and also provide a mechanism to track any malicious activity.

Algorithm 3: Łukasiewicz Fuzzy Smart Contract Verification

Input: User ' $\mathrm{U}=\mathrm{B}, \mathrm{M}, \mathrm{TA}, \mathrm{IA}, \mathrm{D}$ ', Buyer ' $\mathrm{B}=\mathrm{B}_{1}, \mathrm{~B}_{2}, \ldots, \mathrm{B}_{\mathrm{n}}$ ' Manufacturer (different make) ' $\mathrm{M}=\mathrm{M}_{1}, \mathrm{M}_{2}, \ldots, \mathrm{M}_{\mathrm{n}}$ ', Transport Authority 'TA', Insurance Authority ' $\mathrm{IA}=\mathrm{IA}_{1}, \mathrm{IA}_{2}, \ldots, \mathrm{IA}_{\mathrm{n}}$ ', Dealer 'D $=\mathrm{d}_{1}, \mathrm{~d}_{2}, \ldots, \mathrm{d}_{\mathrm{n}}$ ', feature ' $\mathrm{f}$ ', Cloud Service Provider 'CSP' 
Output: Accurate and precise prediction of fraudulent activities

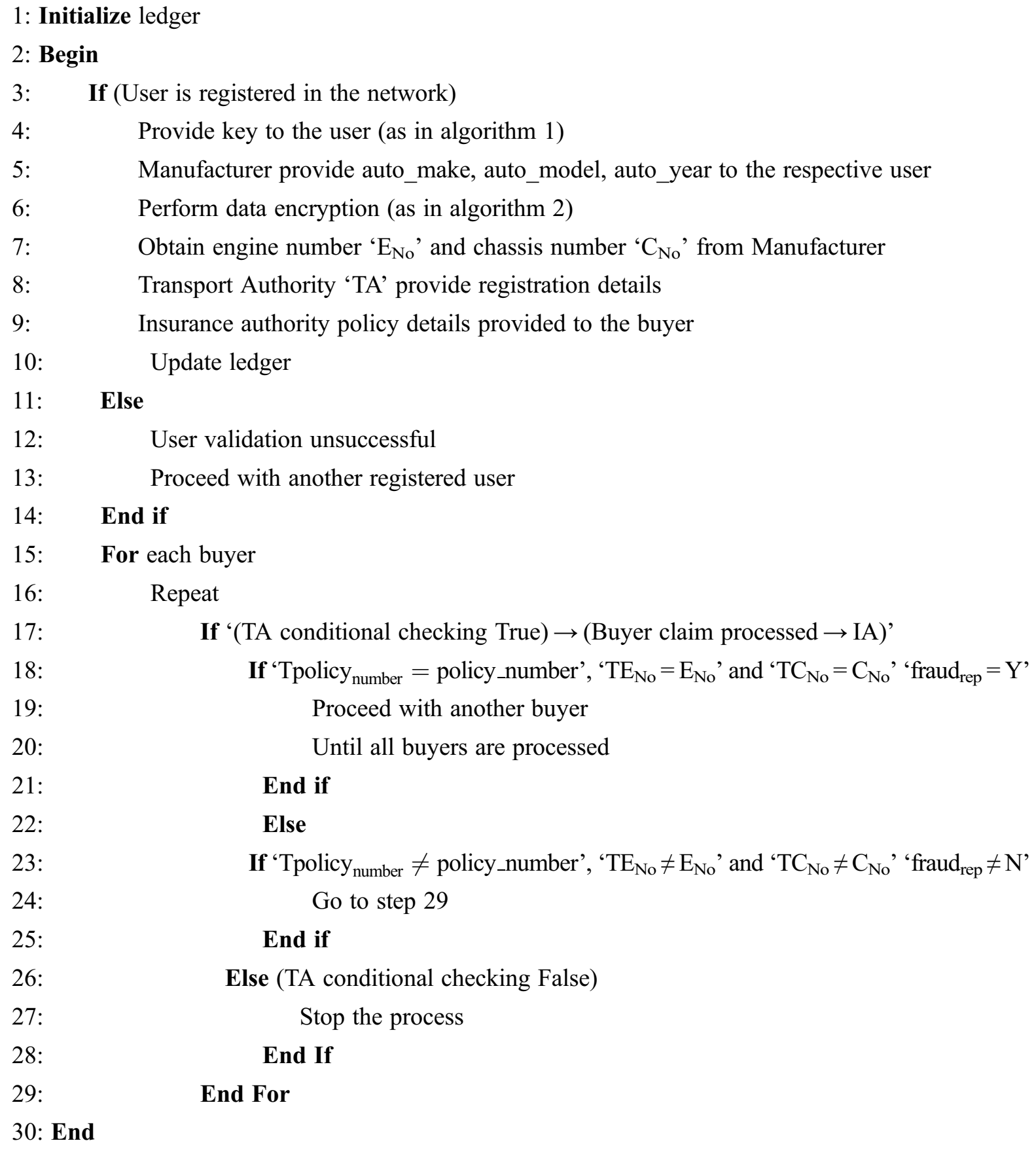




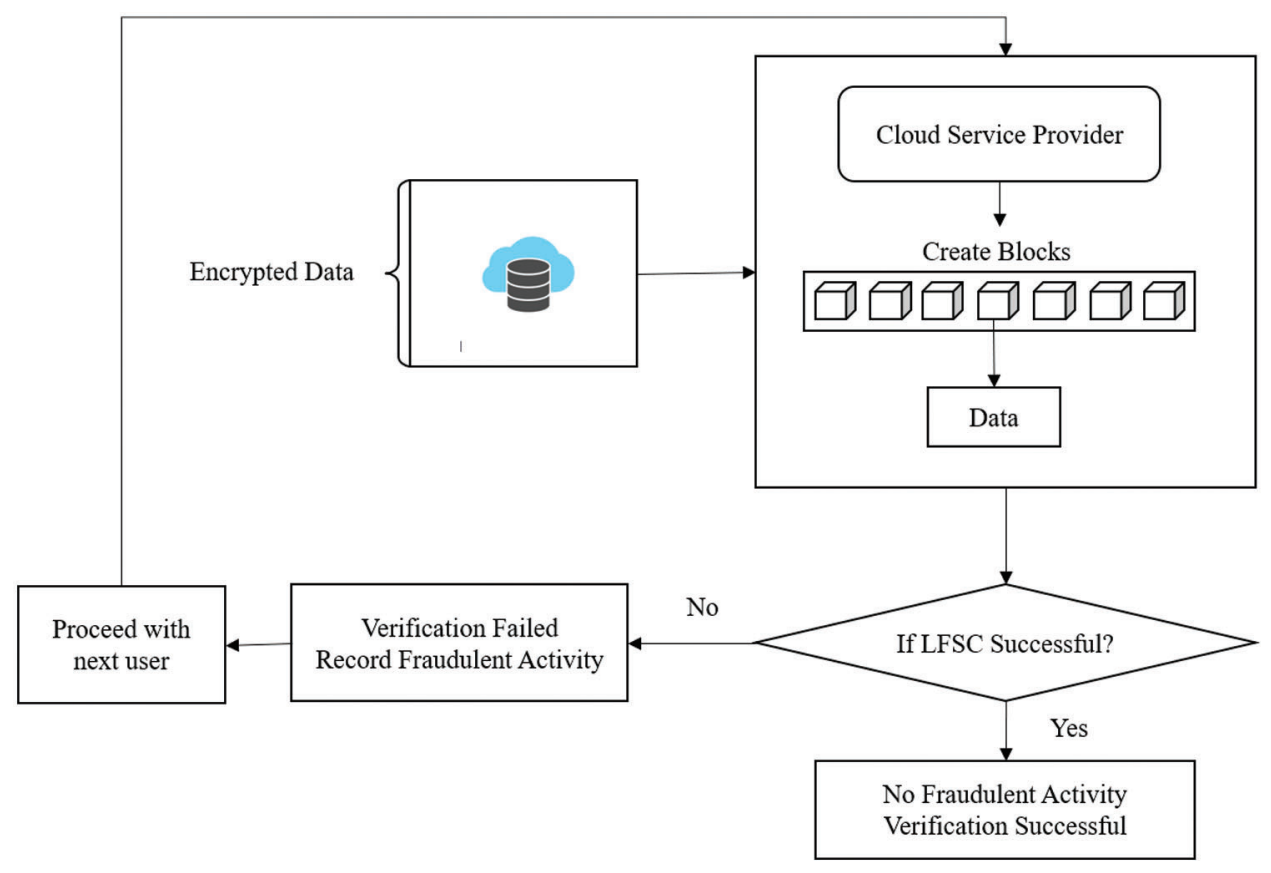

Figure 4: Block diagram of Łukasiewicz fuzzy smart contract verification model

\section{Results and Discussion}

This section presents a comparative analysis and discussion of the vehicle life cycle tracking method proposed in this work to explore the efficiency of the proposed method in terms of tracking time, tracking overhead, and tracking accuracy based on the auto insurance claims dataset from Kaggle. The dataset includes 40 columns. To mention a few are month_as_customer, age, policy_number, policy_bind_date, policy_status, insured_sex, insured_zip, incident_state, incident_date, property_claim, vehicle_claim, auto_make, auto_model, auto_year and so on. JAVA platform with CloudSim simulation was used to simulate the techniques and bring out the effectiveness of the method. The CloudSim is a Framework for Modeling and Simulation of Cloud Computing Infrastructures and Services. The parameters used in the simulation are the number of users, tracking time, and memory consumption. The simulation is carried out for $30 \mathrm{~min}$, and the number of users was varied from 50 to 500 .

\subsection{Performance Evaluation of Vehicle Life Cycle Tracking Time}

One of the significant parameters is the vehicle life cycle tracking time. Lesser the vehicle life cycle tracking time more efficient the vehicle fleet management is said to be. In other words, the vehicle life cycle tracking time refers to the time consumed in tracking the vehicle life cycle. It is mathematically evaluated using Eq. (10).

$$
\mathrm{VLCT}_{\text {time }}=\mathrm{U}_{\mathrm{i}} * \text { Time }[\mathrm{VLCT}]
$$

From Eq. (10), the vehicle life cycle tracking time ' $\mathrm{VLCT}_{\text {time }}$ ' is measured based on the number of users ' $\mathrm{U}_{\mathrm{i}}$ ' and the time consumed in tracking the vehicle life cycle 'Time VLCT'. It is evaluated in terms of milliseconds (ms). Fig. 5 and Tab. 1 show the vehicle life cycle tracking time for a different number of users obtained from the auto insurance claims dataset. The vehicle life cycle tracking time increases with the number of users involved in the simulation. As the number of users increases, authentication and registration involved also increases, therefore increasing the vehicle life cycle tracking time also. From the results, it is inferred that the vehicle life cycle tracking time was found to be comparatively lesser 
using the proposed HOGD-LF upon comparison with two other existing methods. The reason behind the improvement was the application of the Progressive Harmonic Search Optimization (PHSO) algorithm. By applying this algorithm, computationally efficient keys are generated. Harmony Memory Considering Rate and Pitching Adjust Rate helps to obtain enhanced candidate solutions. The vehicle life cycle tracking time has been reduced using HOGD-LF by 6\% compared to the framework proposed by Syed et al., and 35\% compared to the framework proposed by Yang et al., respectively.

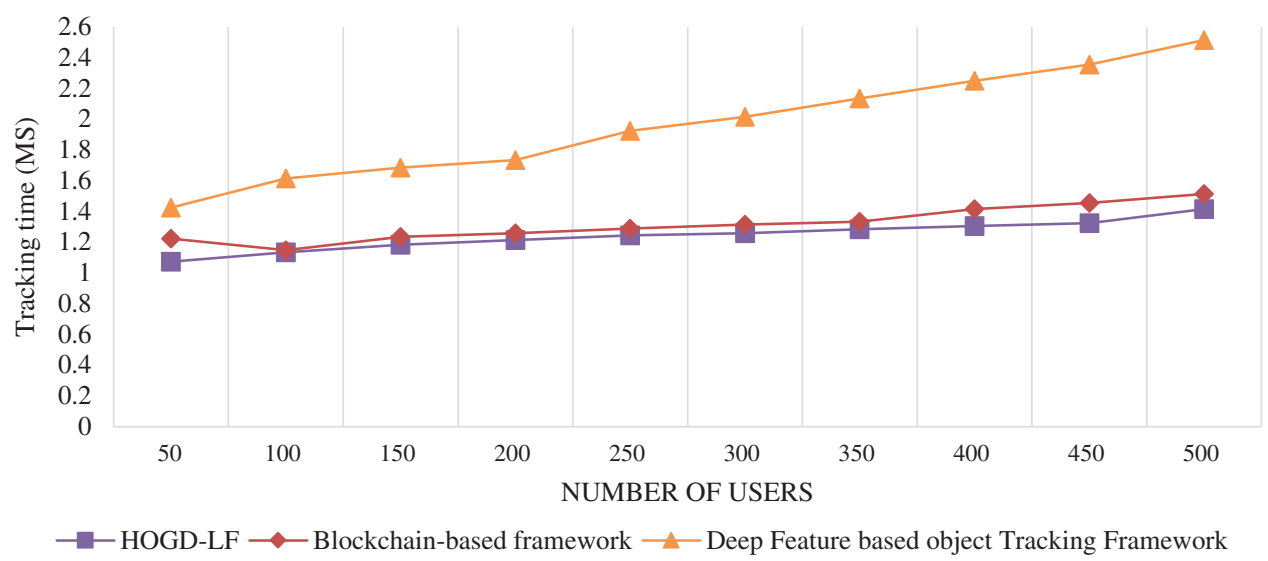

Figure 5: Graphical representation of vehicle life cycle tracking time

Table 1: Evaluation of tracking time

\begin{tabular}{llll}
\hline Number of users & \multicolumn{2}{c}{ Vehicle life cycle tracking time (ms) } \\
\cline { 2 - 4 } & HOGD-LF & $\begin{array}{l}\text { Blockchain-based } \\
\text { framework }\end{array}$ & $\begin{array}{l}\text { Deep feature based object } \\
\text { tracking framework }\end{array}$ \\
\hline 50 & 1.075 & 1.225 & 1.425 \\
100 & 1.135 & 1.15 & 1.615 \\
150 & 1.185 & 1.235 & 1.685 \\
200 & 1.215 & 1.26 & 1.735 \\
250 & 1.245 & 1.29 & 1.925 \\
300 & 1.26 & 1.315 & 2.015 \\
350 & 1.285 & 1.335 & 2.135 \\
400 & 1.305 & 1.415 & 2.25 \\
450 & 1.325 & 1.455 & 2.355 \\
500 & 1.415 & 1.515 & 2.515 \\
\hline
\end{tabular}




\subsection{Performance Evaluation of Vehicle Life Cycle Tracking Overhead}

The next parameter of significance involved in vehicle life cycle tracking is the overhead involved in vehicle life cycle tracking. This is because of the reason that during tracking a small amount of overhead is said to occur. This is mathematically formulated as given below.

$\mathrm{VLCT}_{\mathrm{OH}}=\mathrm{U}_{\mathrm{i}} * \mathrm{Mem}[\mathrm{VLCT}]$

From Eq. (11), the vehicle life cycle tracking overhead ' $\mathrm{VLCT}_{\mathrm{OH}}$ ' is measured based on the number of users involved in simulation ' $U_{i}$ ' and the memory consumed in tracking the vehicle life cycle 'Mem VLCT'. It is measured in terms of kilobytes (KB). Fig. 6 and Tab. 2 illustrate the vehicle life cycle tracking overhead for different users in the range of 50 to 500. Increasing the number of users causes a significant increase in the overhead also. The reason behind the increase is that the increased number of users involved in vehicle life cycle tracking, a large number of users has to be authenticated, and therefore overhead for the encryption mechanism to be applied also increases. From the results, it is inferred that the overhead involved in vehicle life cycle tracking is substantially improved in the proposed HOGD-LF method. The reason behind the reduction in overhead is due to the incorporation of the Gradient Descent Blockchain-based Data Encryption (GDB-DE) algorithm. Gradient Descent function helps in the efficient generation of keys for encryption and decryption. This in turn reduces the overhead involved in vehicle life cycle tracking using HOGD-LF by 33\% and $48 \%$ upon comparison with two other methods proposed by Syed et al., and Yang et al., respectively.

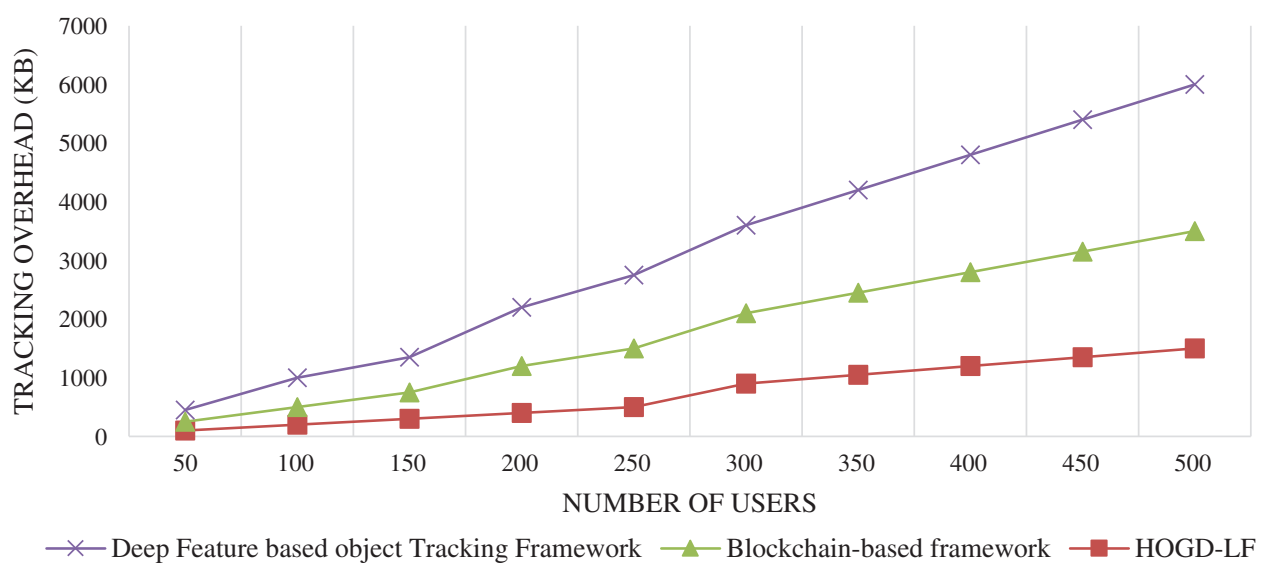

Figure 6: Graphical representation of vehicle life cycle tracking overhead

\subsection{Performance Evaluation of Vehicle Life Cycle Tracking Accuracy}

The vehicle life cycle tracking accuracy is estimated to bring out the efficiency of the work. This is mathematically formulated as given below.

$\mathrm{VLFC}_{\mathrm{acc}}=\sum_{\mathrm{i}=1}^{\mathrm{n}} \frac{\mathrm{U}_{\mathrm{CT}}}{\mathrm{U}_{\mathrm{i}}} * 100$

As shown in Eq. (12), the vehicle life cycle tracking accuracy ' $V L F C$ acc is measured based on the number of users involved in simulation setup ' $\mathrm{U}_{\mathrm{i}}$ ' and the users or vehicles correctly tracked ' $\mathrm{U}_{\mathrm{CT}}$ '. It is measured in terms of percentage (\%). Fig. 7 and Tab. 3 present the comparative analysis of three techniques in terms of accuracy. It can be inferred that the proposed technique performs better than the existing approaches in terms of accuracy. It can be observed that the increase in the number of users causes a gradual decrease in the accuracy percentage. With the increase in the number of users, a significant number of users being 
correctly tracked is also reduced. The overall vehicle life cycle tracking accuracy being ' $84 \%$ ', ' $82 \%$ ', and ' $80 \%$ ' using the proposed HOGD-LF and two existing methods. From the results, it is evident that the accuracy obtained using HOGD-LF is improved by 5\% compared to the framework proposed by Syed et al., and 10\% compared to the framework proposed by Yang et al. respectively. This is achieved due to the application of the Lukasiewicz Fuzzy Smart Contract algorithm in which data accesses and data modification is performed simultaneously. Besides, information about the place and unique ID of the user is also stored in the corresponding block.

Table 2: Evaluation of tracking overhead

\begin{tabular}{llll}
\hline Number of users & & Vehicle life cycle tracking overhead (KB) \\
\cline { 2 - 4 } & HOGD-LF & $\begin{array}{l}\text { Blockchain-based } \\
\text { framework }\end{array}$ & $\begin{array}{l}\text { Deep feature based } \\
\text { object tracking framework }\end{array}$ \\
\hline 50 & 100 & 150 & 200 \\
100 & 200 & 300 & 500 \\
150 & 300 & 450 & 600 \\
200 & 400 & 800 & 1000 \\
250 & 500 & 1000 & 1250 \\
300 & 900 & 1200 & 1500 \\
350 & 1050 & 1400 & 1750 \\
400 & 1200 & 1600 & 2000 \\
450 & 1350 & 1800 & 2250 \\
500 & 1500 & 2000 & 2500 \\
\hline
\end{tabular}

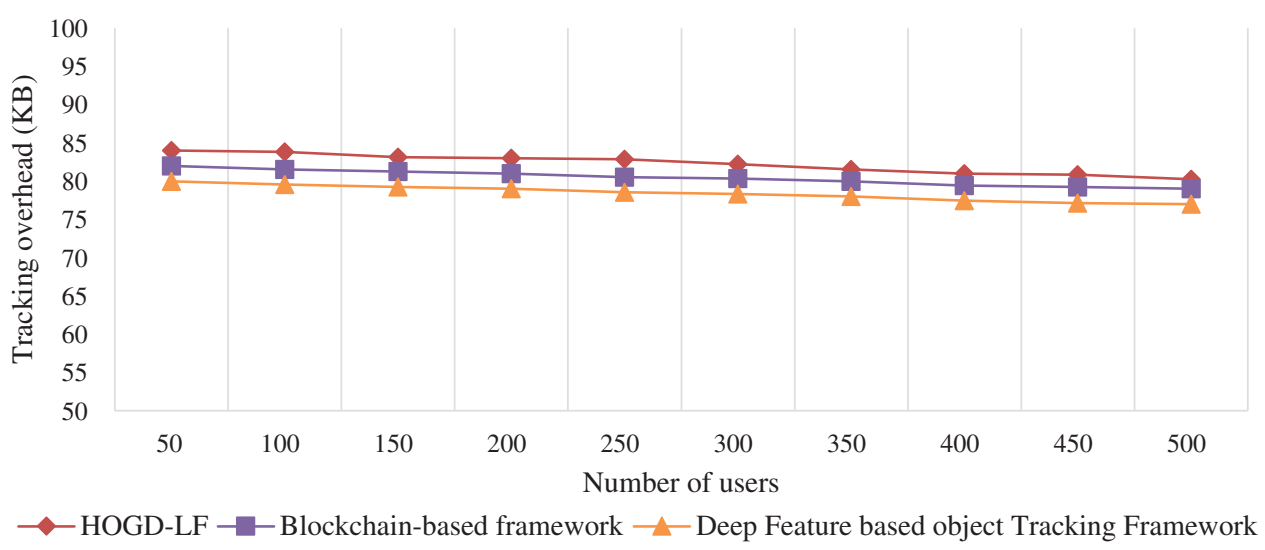

Figure 7: Graphical representation of vehicle life cycle tracking accuracy 
Table 3: Estimation of tracking accuracy

\begin{tabular}{llll}
\hline Number of users & \multicolumn{3}{c}{ Vehicle life cycle tracking accuracy (\%) } \\
\cline { 2 - 4 } & HOGD-LF & $\begin{array}{l}\text { Blockchain-based } \\
\text { framework }\end{array}$ & $\begin{array}{l}\text { Deep feature-based } \\
\text { object tracking framework }\end{array}$ \\
\hline 50 & 84 & 82 & 80 \\
100 & 83.85 & 81.55 & 79.55 \\
150 & 83.15 & 81.25 & 79.25 \\
200 & 83 & 81 & 79 \\
250 & 82.85 & 80.55 & 78.55 \\
300 & 82.25 & 80.35 & 78.35 \\
350 & 81.55 & 80 & 78 \\
400 & 81 & 79.45 & 77.45 \\
450 & 80.85 & 79.25 & 77.15 \\
500 & 80.25 & 79 & 77 \\
\hline
\end{tabular}

\section{Conclusion and Future Scope}

A solution to enhance the existing vehicle lifecycle network is proposed. The proposed framework makes use of blockchain technology to enforce confidentiality and trust. It provides secure sharing of data among various stakeholders. In this work, a blockchain-based framework for Vehicle Life Cycle Tracking in Cloud Environment, Harmonic Optimized Gradient Descent, and Lukasiewicz Fuzzy (HOGD-LF) is proposed for timely and precise tracking. The proposed method consists of registration \& authentication, data encryption, and smart contract-based verification algorithms which help in reducing the detection time, memory overhead and improving the tracking accuracy. PHSO algorithm uses progressive functions for both HMCR and PAR to ensure computationally efficient key generation for user registration and authentication. The GDB-DE algorithm combines machine learning with the blockchain network to achieve better performance by reducing time, memory overhead, increasing accuracy, and improve CPU utilization. The LFSC algorithm ensures security and provides a mechanism to track any malicious activity. The experimental evaluation revealed the effectiveness of the proposed method with better performance in terms of vehicle life cycle tracking time, overhead and accuracy. The tracking time is reduced by $6 \%$ and $35 \%$, the memory overhead is reduced by $33 \%$ and $48 \%$ and finally, the tracking accuracy is improved by $5 \%$ and $10 \%$ in comparison to the two techniques available in the literature.

Blockchain technology is continuously evolving. The inclusion of vehicle dashboard information and updating them frequently will help in achieving higher tracking accuracy. The use of side chains and multiple blockchain networks for online and offline status tracking will help to improve the overall system efficiency and reduce frequent data upload to the cloud. The work can also be enhanced by considering the scalability issues and expanding the range of participants who can utilize this network. 
Acknowledgement: The authors wish to express their sincere thanks to the Department of Science \& Technology, New Delhi, India (Project ID: SR/FST/ETI-371/2014) and express their sincere thanks to the INSPIRE fellowship (DST/INSPIRE Fellowship/2016/IF160837) for their financial support. The authors also thank SASTRA Deemed to be University, Thanjavur, India for extending the infrastructural support to carry out this work.

Funding Statement: The authors have not received any specific funding for this research work.

Conflicts of Interest: The authors declare that they have no conflicts of interest to report regarding the present study.

\section{References}

[1] S. A. Abeyratne and M. P. Radmehr, "Blockchain ready manufacturing supply chain using distributed ledger," International Journal of Research in Engineering and Technology, vol. 5, no. 9, pp. 1-10, 2016.

[2] T. A. Syed, M. S. Siddique, A. Nadeem, A. Alzahrani, S. Jan et al., "A novel blockchain-based framework for vehicle life cycle tracking: An end-to-end solution," IEEE Access, vol. 8, no. June, pp. 111042-111063, 2020.

[3] B. Yang, M. Tang, S. Chen, G. Wang, Y. Tan et al., "A vehicle tracking algorithm combining detector and tracker," EURASIP Journal on Image and Video Processing, vol. 2020, no. 1, pp. 17, 2020.

[4] T. Ali Syed, A. Alzahrani, S. Jan, M. S. Siddiqui, A. Nadeem et al., "A comparative analysis of blockchain architecture and its applications: Problems and recommendations," IEEE Access, vol. 7, no. 6, pp. 176838176869, 2019.

[5] A. Zhang, R. Y. Zhong, M. Farooque, K. Kang and V. G. Venkatesh, "Blockchain-based life cycle assessment: An implementation framework and system architecture," Resources, Conservation and Recycling, vol. 152, no. May 2019, pp. 104512, 2020.

[6] N. Elisa, L. Yang, F. Chao and Y. Cao, "A framework of blockchain-based secure and privacy-preserving Egovernment system," Wireless Networks, vol. 1, pp. 1-11, 2018.

[7] K. L. Brousmiche, T. Heno, C. Poulain, A. Dalmieres and E. B. Hamida, "Digitizing, securing and sharing vehicles life-cycle over a consortium blockchain: Lessons learned," in 2018 9th IFIP Int. Conf. on New Technologies, Mobility and Security, NTMS 2018-Proc., Paris, France, vol. 2018-Janua, pp. 1-5, 2018.

[8] P. K. Sharma, N. Kumar and J. H. Park, "Blockchain-based distributed framework for automotive industry in a smart city," IEEE Transactions on Industrial Informatics, vol. 15, no. 7, pp. 4197-4205, 2019.

[9] S. O. Lee, H. Jung and B. Han, "Security assured vehicle data collection platform by blockchain: Service provider's perspective," in Int. Conf. on Advanced Communication Technology, ICACT, Pyeong Chang Kwangwoon_Do, Korea (South), vol. 2019-Febru, pp. 265-268, 2019.

[10] I. Makhdoom, M. Abolhasan, H. Abbas and W. Ni, "Blockchain's adoption in IoT: The challenges, and a way forward," Journal of Network and Computer Applications, vol. 125, pp. 251-279, 2019.

[11] M. Demir, O. Turetken and A. Ferworn, "Blockchain based transparent vehicle insurance management," in 2019 6th Int. Conf. on Software Defined Systems, SDS 2019, Rome, Italy, pp. 213-220, 2019.

[12] P. K. Sharma, S. Y. Moon and J. H. Park, "Block-vN: A distributed blockchain based vehicular network architecture in smart city," Journal of Information Processing Systems, vol. 13, no. 1, pp. 184-195, 2017.

[13] J. Stodt, E. Jastremskoj, C. Reich, D. Welte and A. Sikora, "Formal description of use cases for industry 4.0 maintenance processes using blockchain technology," Proc. of the 2019 10th IEEE Int. Conf. on Intelligent Data Acquisition and Advanced Computing Systems: Technology and Applications, IDAACS 2019, Lorraine, France, vol. 2, pp. 1136-1141, 2019.

[14] T. Reimers, F. Leber and U. Lechner, "Integration of blockchain and internet of things in a car supply chain," in Proc.-2019 IEEE Int. Conf. on Decentralized Applications and Infrastructures, DAPPCON 2019, California, USA, pp. 146-151, 2019.

[15] A. Nordelöf, "A scalable life cycle inventory of an automotive power electronic inverter unit-part II: Manufacturing processes," International Journal of Life Cycle Assessment, vol. 24, no. 4, pp. 694-711, 2019. 
[16] T. Su, S. Shao, S. Guo and M. Lei, "Blockchain-based internet of vehicles privacy protection system," Wireless Communications and Mobile Computing, vol. 2020, pp. 1-10, 2020.

[17] A. Del Duce, M. Gauch and H. J. Althaus, "Electric passenger car transport and passenger car life cycle inventories in ecoinvent version 3," International Journal of Life Cycle Assessment, vol. 21, no. 9, pp. 13141326, 2016.

[18] S. Hayrutdinov, M. S. R. Saeed and A. Rajapov, "Coordination of supply chain under blockchain system-based product lifecycle information sharing effort," Journal of Advanced Transportation, vol. 2020, pp. 1-10, 2020.

[19] C. Ranjeeth Kumar and R. Anuradha, "Feature selection and classification methods for vehicle tracking and detection," Journal of Ambient Intelligence and Humanized Computing, vol. 12, no. 3, pp. 4269-4279, 2021.

[20] B. Sen, M. Kucukvar, N. C. Onat and O. Tatari, "Life cycle sustainability assessment of autonomous heavy-duty trucks," Journal of Industrial Ecology, vol. 24, no. 1, pp. 149-164, 2020.

[21] R. Roriz and J. L. Pereira, "Avoiding insurance fraud: A blockchain-based solution for the vehicle sector," Procedia Computer Science, vol. 164, pp. 211-218, 2019.

[22] L. R. Abbade, F. M. Ribeiro, M. H. Da Silva, A. F. P. Morais, E. S. De Morais et al., "Blockchain applied to vehicular odometers," IEEE Network, vol. 34, no. 1, pp. 62-68, 2020.

[23] Y. Yu, Y. Zhao, Y. Li, X. Du, L. Wang et al., "Blockchain-based anonymous authentication with selective revocation for smart industrial applications," IEEE Transactions on Industrial Informatics, vol. 16, no. 5, pp. 3290-3300, 2020.

[24] P. Ahmadi, X. M. Cai and M. Khanna, "Multicriterion optimal electric drive vehicle selection based on lifecycle emission and lifecycle cost," International Journal of Energy Research, vol. 42, no. 4, pp. 1496-1510, 2018.

[25] A. Gomaa, M. M. Abdelwahab and M. Abo-Zahhad, "Efficient vehicle detection and tracking strategy in aerial videos by employing morphological operations and feature points motion analysis," Multimedia Tools and Applications, vol. 79, no. 35-36, pp. 26023-26043, 2020.

[26] H. J. Lee, B. Moon and G. Kim, "Hierarchical scheme of vehicle detection and tracking in nighttime urban environment," International Journal of Automotive Technology, vol. 19, no. 2, pp. 369-377, 2018. 